\title{
Impacto por huracanes en las praderas de Thalassia testudinum (Hydrocharitaceae) en el Caribe Mexicano
}

\author{
Leonardo U. Arellano-Méndez, María de los Ángeles Liceaga-Correa, Jorge A. Herrera-Silveira \\ \& Héctor Hernández-Núñez \\ * CINVESTAV-IPN, Unidad Mérida. Antigua carretera a Progreso Km.6, Mérida, Yucatán. C.P. 97310, México; \\ leonardo@mda.cinvestav.mx, liceaga@mda.cinvestav.mx,jherrera@mda.cinvestav.mx,hnunez@mda.cinvestav.mx
}

Recibido 30-IV-2010. Corregido 30-IX-2010. Aceptado 29-X-2010.

\begin{abstract}
Hurricane impact on Thalassia testudinum (Hydrocharitaceae) beds in the Mexican Caribbean. Hurricanes have increased in strength and frequency as a result of global climate change. This research was conducted to study the spatio-temporal distribution and changes of Thalassia testudinum, the dominant species in Bahia de la Ascension (Quintana Roo, Mexico), when affected by heavy weather conditions. To complete this objective, a 2001 Landsat ETM+ image and the information from 525 sampling stations on morpho-functional and coverage of T. testudinum were used, and the seeds generated for the classification of eight benthic habitats. To quantify the changes caused by two hurricanes, we used two images, one of 1988 (Gilberto) and another of 1995 (Roxanne); other three data sets (2003, 2005 and 2007) were also used to describe the study area without major weather effects. Six categorial maps were obtained and subjected to analysis by 8 Landscape Ecology indexes, that describe the spatial characteristics, structure, function, change of the elements (matrix-patch-corridor), effects on ecosystems, connectivity, edges, shape and patch habitat fragmentation. Models indicate that T. testudinum may be classified as a continuum (matrix), since the fragments were not observed intermittently, but as a progression from minimum to maximum areas in reference to their coverage (ecological corridors). The fragments do not have a regular shape, indicating that the impacts are recent and may be due to direct effects (high-intensity hurricanes) or indirect (sediment). Fragments of type "bare soils" have a discontinuous distribution, and are considered to be the sites that have remained stable over a long timescale. While more dense coverage areas ("beds", "medium prairie" and "prairie") have low fragmentation and high connection of fragments. Features have an irregular perimeter and radial growth of formal; suggesting that the impact of meteors has no effect on the resilience of $T$. testudinum in this ecosystem, indicating good environmental quality to grow in this bay. Rev. Biol. Trop. 59 (1): 385-401. Epub 2011 March 01.
\end{abstract}

Key words: Thalassia testudinum, global climate change, hurricanes, landscape ecology, Landsat ETM+, indexes, resilience, fragmentation coverage.

El Cambio Climático Global (CCG) inducido de manera antrópica promueve cambios de profunda implicación en los ecosistemas marinos, un ejemplo de ello es el efecto de las tormentas tropicales y huracanes en los ecosistemas costeros (Michot et al. 2002, Heck Jr. \& Byron 2005, Sheik 2005), raramente son eventos repetidos o ecológicamente predecibles. Los huracanes pueden inducir cambios en los hábitats terrestres y marinos, y juegan un rol importante en el clima del mundo, al transportar el exceso de calor y humedad de los trópicos, a latitudes templadas y boreales, además alteran el clima local a mediano plazo, resultado de la modificación de la cobertura vegetal y la evapotranspiración (Lugo 2000).

Emanuel (2005) y Webster et al. (2005), demostraron que el incremento en la intensidad de los huracanes se haya asociada con el incremento de la temperatura superficial en el trópico. Webster et al. (2005) examinaron con datos de satélite a nivel global, la actividad de 
los huracanes desde 1970, concluyendo que el promedio del número de huracanes no ha incrementado de manera global, sino el porcentaje de la categoría de 4 y 5 , que ha crecido aproximadamente al doble. Adicionalmente, los ciclones tropicales no se forman a menos que la temperatura del agua exceda $\operatorname{los} 26.5^{\circ} \mathrm{C}$, de tal manera que existe una relación entre la variación en la temperatura tropical superficial y las regiones donde se forman las tormentas ciclónicas tropicales, se observa que en cada una de estas regiones se ha tenido un incremento de $0.5^{\circ} \mathrm{C}$ desde 1970 .

En las regiones tropicales, las comunidades de pastos marinos son impactadas por eventos que alteran su cobertura, composición de especies, biomasa y características de la comunidad, producen su fragmentación y forman parches (Aguayo 2004). Al estar ubicadas en áreas de transición, será ahí donde se observe, de manera muy marcada, el impacto del CCG (Kennish 2002, Dolbeth et al. 2007, Lillebø et al. 2005, Paerl 2006).

Los pastos marinos han sido usados como indicadores ecológicos de estrés natural (huracanes, descargas de agua dulce por lluvias), o antrópico puntual (descargas de aguas contaminadas, deforestación de zonas de manglar), o a un nivel más general (CCG). Esto, debido a que son organismos que poseen una gran sensibilidad a los cambios en la calidad del agua (definidos como la cuantificación de parámetros físicos, químicos y biológicos; Aranda 2001, Arellano-Méndez 2004), además de que son considerados una comunidad clave de los ecosistemas costeros y una parte esencial en sus funciones ecológicas (Zieman 1982, Zieman et al. 1999).

Thalassia testudinum es la especie dominante por distribución y biomasa en Bahía de la Ascensión; a menudo se encuentra mezclada con otras especies de pastos como Halodule wrightii en la parte interna de la boca, central y suroeste; con Syringodium filiforme, en la zona externa de la boca (frente oceánico) y con Ruppia maritima en los sitios de descarga de agua dulce al fondo de la bahía. También se asocia con macroalgas (54 Rhodophytas,
7 Phaeophytas, 40 Chlorophytas), con mayor diversidad de especies hacia el exterior de la bahía y menor hacia el interior; las especies Penicillus capitatus, Bathophora oerstedii, Halimeda incrassata y Laurencia intricata son las más representativas por su abundancia y ocurrencia en el área de estudio (Aguilar et al. 1989, Arellano-Méndez 2004).

Debido a su respuesta como indicador y al ser la especie dominante en Bahía de la Ascensión, se usó a T. testudinum para este estudio, como un indicador de los cambios, que a nivel de paisaje de las diferentes praderas, son producidos por los impactos de dos eventos meteorológicos, el huracán Gilberto (1988) y el huracán Roxanne (1995), eventos de categorías 5 y 3, respectivamente, durante su paso por la bahía.

\section{MATERIALES Y MÉTODOS}

Área de Estudio: El área de estudio es Bahía de la Ascensión (Fig. 1), en la Reserva de la Biosfera de Sian Ka'an, Quintana Roo, México; posee un área de $740 \mathrm{~km}^{2}$, con una profundidad promedio de $3.5 \mathrm{~m}$ y una máxima de $7 \mathrm{~m}$ (Liceaga-Correa 2003), lo que la hace una cuenca somera. La bahía posee un gradiente de salinidad de menores concentraciones en la parte interna (concentración de 1.2) y mayores en su conexión con el mar (hasta 34.2), cuya magnitud varía durante el año (Arellano-Méndez 2004). Tiene una plataforma y una laguna arrecifal frente a la bahía, constituyendo un arrecife de tipo barrera arrecifal.

Material: Se utilizaron seis imágenes Landsat ETM+, una de diciembre 1988 (que corresponde unos meses después del paso del Huracán Gilberto (Fig. 2), una de noviembre 1995, posterior al paso del Huracán Roxanne (Fig. 3), una de abril 2001 (que corresponde al muestreo intensivo), una de abril 2003, una de octubre 2005 y una de diciembre 2007. Estas tres últimas fechas se usaron como parte del programa de monitoreo del área de estudio. A las imágenes se les aplicó correcciones radiométricas, geométricas, atmosféricas y de la columna de agua, y posteriormente fueron 


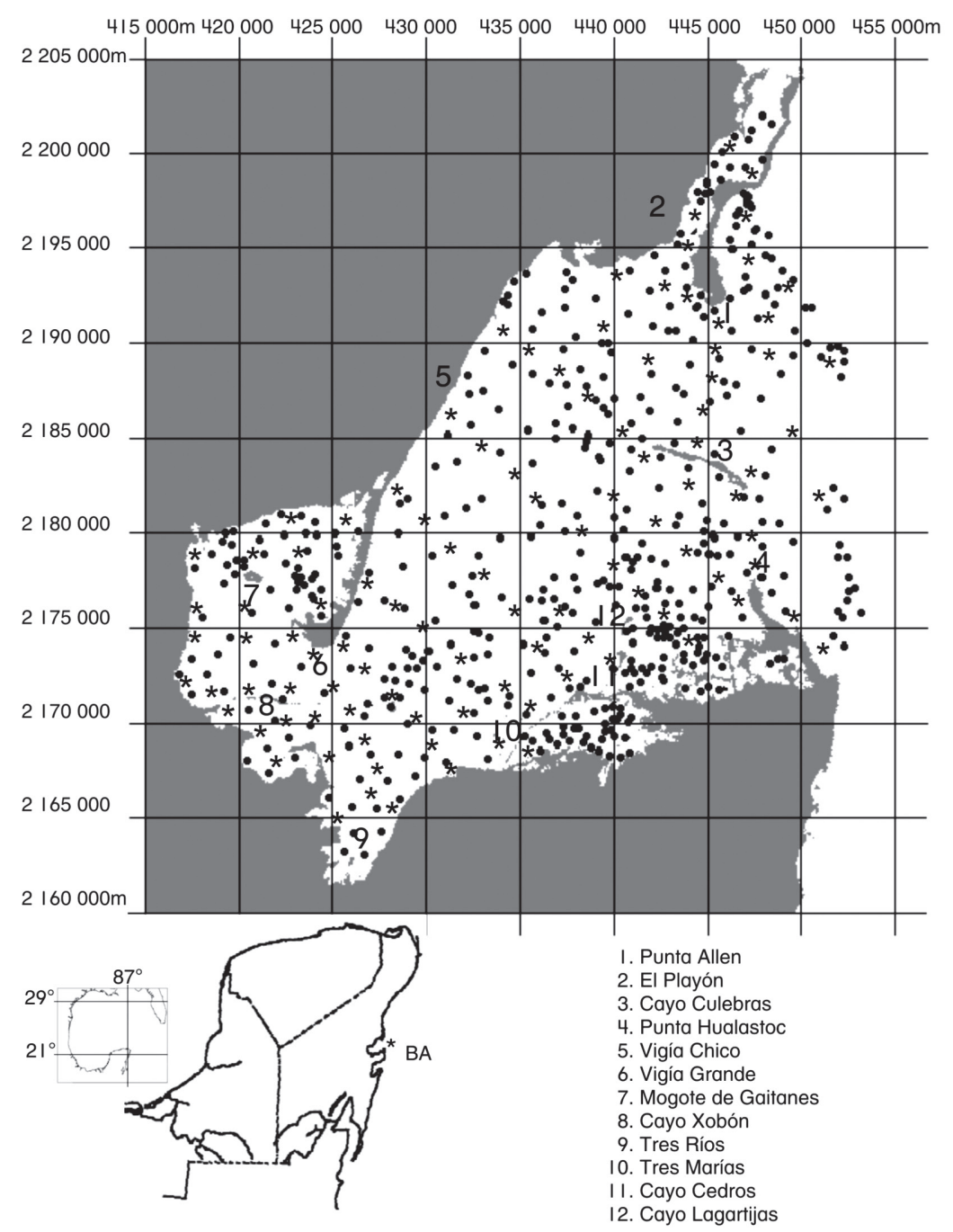

Fig. 1. Bahía de la Ascensión, estaciones de muestreo 2001 (puntos), 2002 a 2007 (cruces) y principales sitios de orientación (números).

Fig. 1. Bahía de la Ascensión, sampling stations 2001 (points), 2002 to 2007 (crosses) and principal orientation places (numbers).

corregistradas las imágenes de 1988, 1995, 2003, 2005 y 2007, con respecto a la imagen del 2001 (path=19, row=46).

Durante el periodo 2000-2001 se realizó el trabajo de campo, en una red de 525 estaciones, y para los años 2003, 2005 y 2007, se tomaron datos en 88 del total de las estaciones como monitoreo del área (Fig. 1). Con el fin de obtener información de los Grandes Grupos
Morfofuncionales (GGMF; tales como corales duros, octocorales, esponjas, pastos marinos, macroalgas), en cada estación de muestreo se tomaron cinco puntos (cuatro esquinas y el centro), cubriendo un área aproximada de 30x30m, de los cuales se estimó la cobertura, abundancia y frecuencia, aplicando el método de Braun Blanquet (Fourqurean et al. 2003); se colocó tres cuadrantes de $1 \times 1 \mathrm{~m}$ al azar en cada 


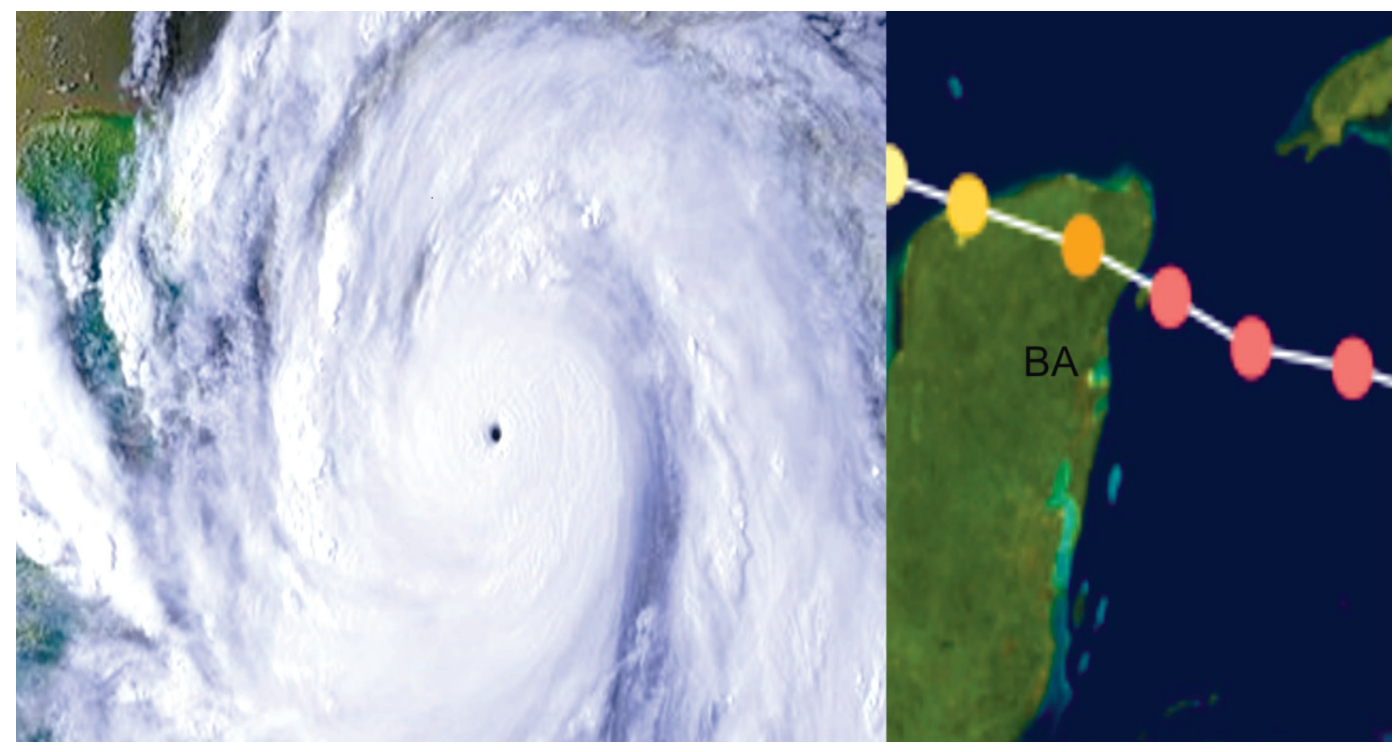

Fig. 2. Huracán Gilberto (categoría 5 en la escala Saffir-Simpson) a su paso por la Península de Yucatán (1986) y parte de la ruta que siguió en el Caribe (Modif. www.nhc.noaa.gov).

Fig. 2. Hurricane Gilberto (category 5 on the Saffir-Simpson scale) as it passes through the Yucatan Peninsula (1986) and part of the route followed in the Caribbean (Modif. www.nhc.noaa.gov).

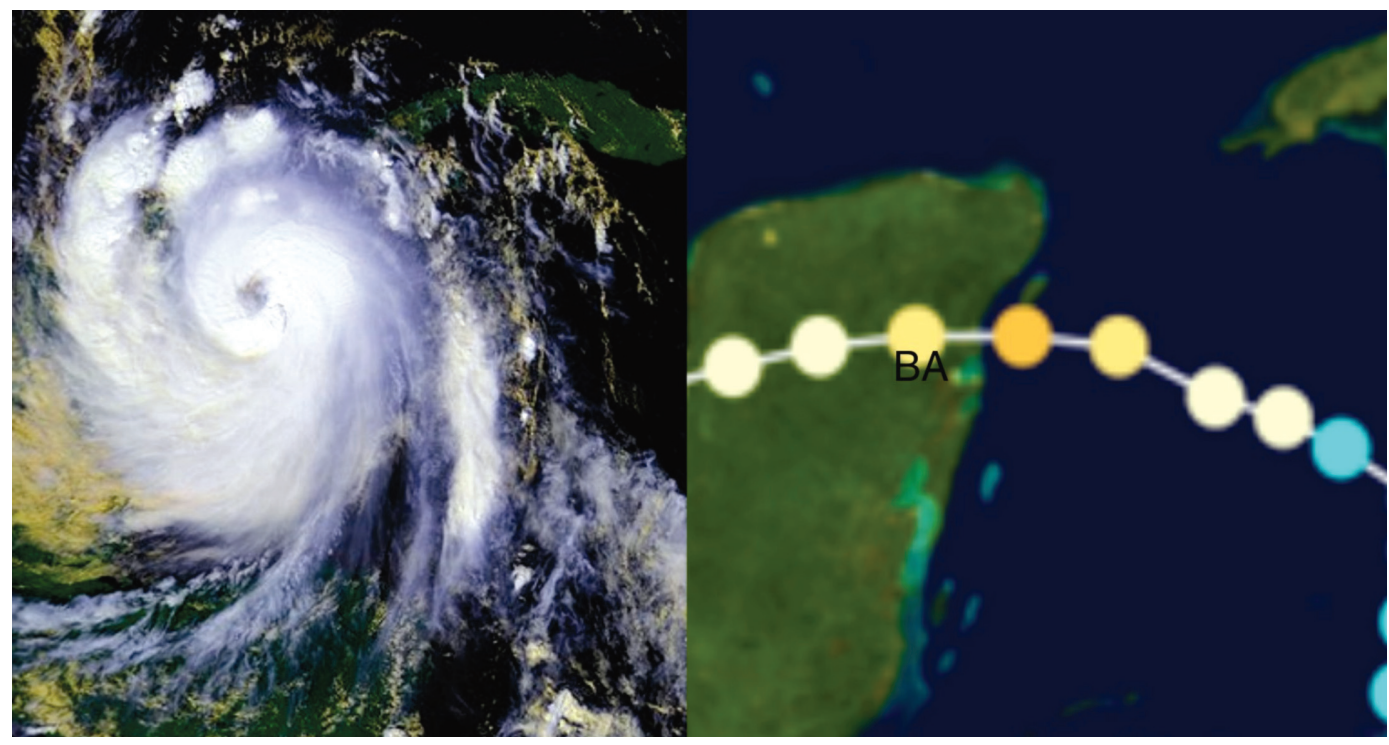

Fig. 3. Huracán Roxanne (categoría 3 en la escala Saffir-Simpson) a su paso por la Península de Yucatán (1993) y parte de la ruta que siguió en el Caribe (Modif. www.nhc.noaa.gov).

Fig. 3. Hurricane Roxanne (category 3 on the Saffir-Simpson scale) as it passes through the Yucatan Peninsula (1993) and part of the route followed in the Caribbean (Modif. www.nhc.noaa.gov). 
uno de los cinco puntos de cada estación. Para cada cuadrante se anotó las especies que se observaron, el grupo al que pertenece cada una y el porcentaje de cobertura, usando la escala recomendada por el método. Para obtener la biomasa aérea, enterrada y densidad de los pastos marinos, se tomó una muestra y su réplica con un nucleador de PVC de $0.35 \mathrm{~m}^{2}$ en cada uno de los cinco sitios de cada estación, aplicando el método de cosecha en pie (standing crop) propuesto por Milner \& Hugues (1968).

Con los datos se generaron las semillas para la clasificación supervisada de los hábitats bénticos (Fig. 4) de la imagen Landsat ETM+ del 21 de abril de 2001 (Liceaga-Correa 2003), se obtuvieron ocho clases, denominadas de acuerdo a las características de los GGMF y a la cobertura de T. testudinum (Clase 1-Barrera Coralina y Colonias de Corales Blandos; Clase 2-Corales Blandos y Macroalgas; Clase 3- Praderas; Clase 4-Praderas Medias; Clase 5-Camas; Clase 6-Praderas; Clase 7-Disperso; Clase 8-Ralo; Clase 9-Organismo Solitario). Con el fin de tener siempre la misma cantidad de hábitats bénticos, se usaron las mismas semillas (Clases) para realizar la clasificación supervisada de las imágenes de 1988 y 1995 que corresponden a años con efecto de huracán de gran intensidad y 2001, 2003, 2005 y 2007, pertenecientes a periodos sin eventos meteorológicos mayores.

Por medio del programa Fragstats (Spatial Patterns Analysis Program for Quantifiying Landscape Structure, Fragstats, Ver. 3.3, McGarigal et al. 2002) se realizó un análisis de patrones espaciales (ecología del paisaje) de los seis mapas categóricos, para definir las medidas (índices) que describieran mejor las características espaciales de cada parche (ocho para nuestro caso: Área Total, Número de Parches, Densidad de Parches, Índice de Tamaño del Parche, Índice de Forma del Paisaje, Índice de Dimensión Fractal, Índice de Cohesión de los Parches), con el fin de observar cambios en los hábitats bénticos, se cuantificó la extensión del área y la configuración espacial de los mosaicos territoriales (parches) en el paisaje.

Adicionalmente, se determinó la estructura (organización espacial de los elementos: matrizmancha-corredor), la función (movimiento o

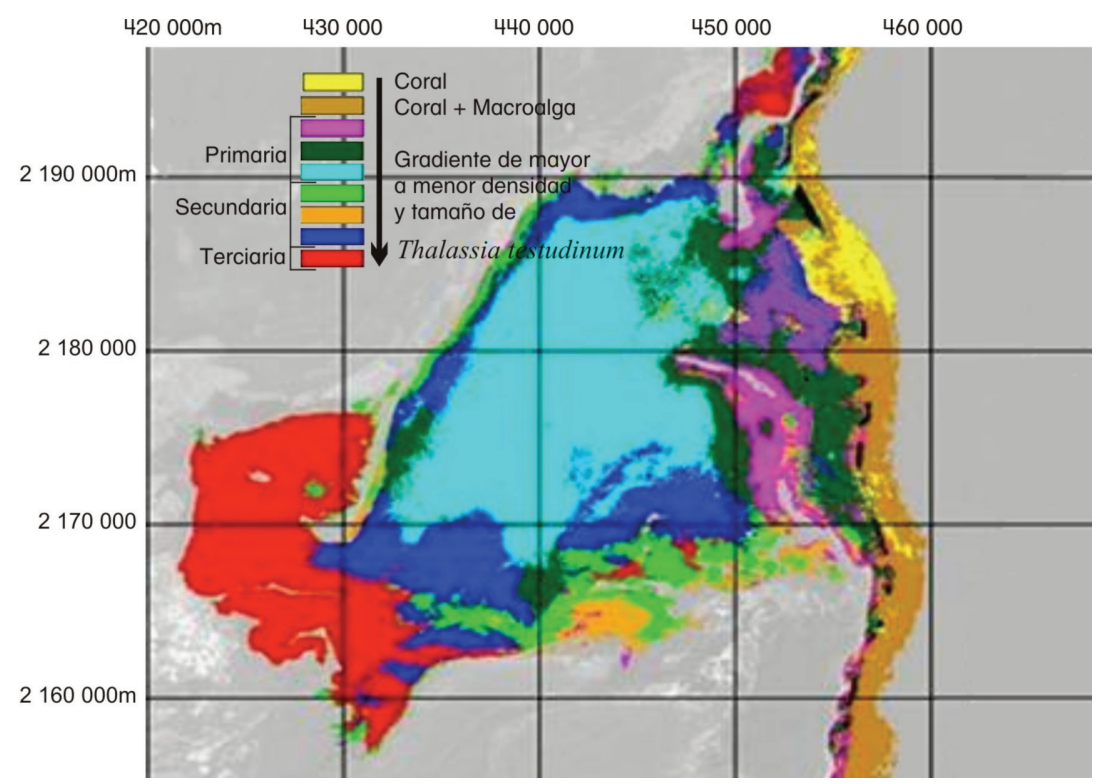

Fig. 4. Hábitats bénticos de Bahía de la Ascensión del año 2001 (Modif. Liceaga-Correa 2003).

Fig. 4. Bahía de la Ascensión bentic habitats from 2001 year (Modif. Liceaga-Correa 2003). 
flujo de materias a través de la estructura) y el cambio (dinámica o transformación del modelo a lo largo del tiempo) de las propiedades de los tres elementos (matriz-mancha-corredor) y sus efectos sobre el ecosistema. Se determinó la conectividad del paisaje, los bordes y formas del parche, así como la fragmentación del hábitat (aumenta el número de manchas, disminuye el tamaño medio de los parches, aumenta el aislamiento de los parches).

\section{RESULTADOS}

El análisis de la composición ecológica del paisaje, reveló que el nivel de escala idóneo para la presente investigación fue a nivel de clases, pues a un nivel mayor (paisaje) o menor (parches), no se establecía un buen resultado, pues a nivel de paisaje se observan los cambios, pero no dónde se producen, mientras que a nivel de parches, son tantas las variaciones presentes que es imposible compararlos entre sí para evaluarlos.

Bahía de la Ascensión posee un área total de $740 \mathrm{~km}^{2}$, más el área de la laguna arrecifal (comprendida fuera de la bahía y hasta la zona del arrecife de barrera) y la ría (entre Punta Allen y "El Playón"), que corresponde a un área aproximada de $85 \mathrm{~km}^{2}$ (Fig. 1). Por su cobertura, la zona que corresponde a la bahía puede dividirse en tres secciones: un área principal asociada con la zona de la boca con la mayor presencia de T. testudinum, donde se denominaron las clases 3-Praderas, 4-Praderas medias y 5-Camas, con características marinas; una secundaria con menor presencia de T. testudinum y las clases 6-Parches, 7-Disperso y 8-Ralo, ubicadas en una zona polihalina, con descargas de agua dulce subterráneas; y una tercera nombrada clase 9-Organismos solitarios, relativamente aislada por una barrera física (un brazo de tierra llamado Vigía Grande), localizada en el fondo de la bahía y de características oligohalinas (Fig. 4).

El resultado del análisis por medio del Fragstats a nivel de clase de los seis mapas categóricos muestra que el área donde se observa dominancia de T. testudinum es en las clases
3 (Praderas), 4 (Praderas Medias) y 5 (Camas), las cuales para el año de 1986 cubrían un área mayor a las $290 \mathrm{~km}^{2}$ (Cuadro 1; Fig. 5) y en la imagen de 1988 había una pérdida de cobertura de $2.58 \mathrm{~km}^{2}$, posiblemente ocasionada por el paso del huracán Gilberto. En 1993, la clase 3 no presentaba signos de recuperación, mientras que las clases 4 y 5 , sí registraron un incremento en el área total de su cobertura y se mostraron casi recuperadas para el 2001 (Cuadro 1, Fig. 5). Es probable que en ese año alcanzará una condición de estabilidad, pues no hubo un aumento notorio del área de cobertura y se registraron variaciones mínimas en los años siguientes de monitoreo, sin embargo, se registró un aumento en el número de parches indicando una alta conectividad (Cuadro 2, Fig. 5).

La recuperación del área total de cobertura de cada clase puede ser debida a que no se afectaron significativamente los parches que componen esas áreas; las clases 3 y 4 han permanecido con una partición mínima de su cobertura, teniéndose para la clase 3 , la pérdida de un $12.43 \%$ de los parches para 1988 , mientras que para 2001 esa pérdida fue de solo un $9.58 \%$, con respecto al área de 1986 . Para 2001, la clase 5 muestra un aumento de $140 \%$ en su número de parches, pero no en su área, indicando que los parches se han fragmentado. Estos resultados indican que los parches se han fragmentado en especial las clases 4 y 5 , ésta relación puede observarse en el Índice de Tamaño del Parche, siendo éstos valores $=>1$, por lo que el incremento en el tamaño de los parches es de manera lenta, lo que sugirie que los factores físicos los afectan de forma importante. Esta división puede deberse a la acción de chorros intensos de agua que impactan de manera dispar a lo largo de las praderas de pastos generando claros sin vegetación. En el ciclo de toma de muestras posterior, se observa que dicha fragmentación se encuentra en recuperación, pues el número de parches disminuye en un $16.65 \%$ de 2003 a 2007, no así el área total de cobertura (Cuadro 2, Fig. 5)

La sección secundaria que corresponde a las clases 6,7 y 8 , poseían en conjunto un área de $230 \mathrm{~km}^{2}$ en 1986 , viéndose disminuidas 


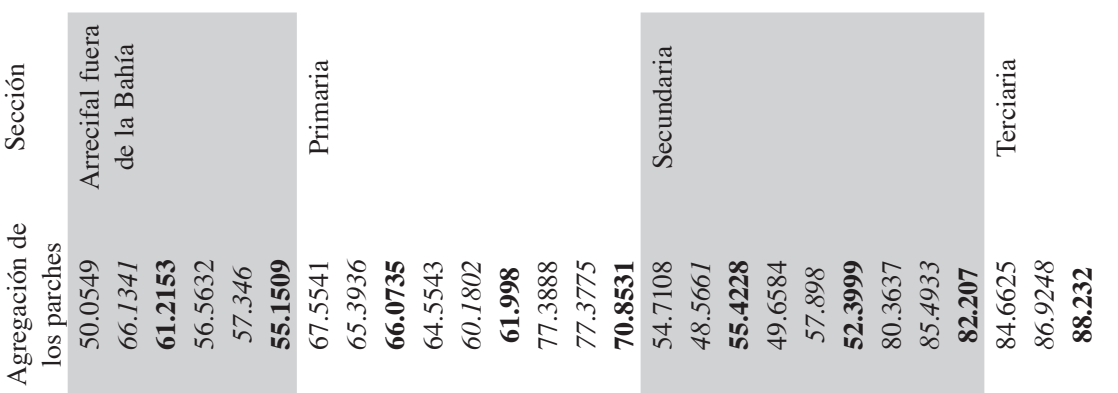

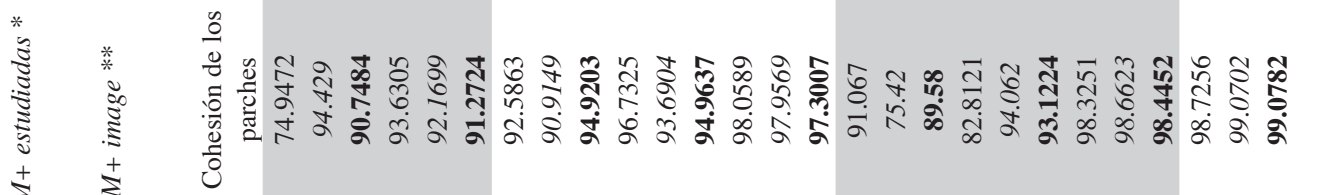

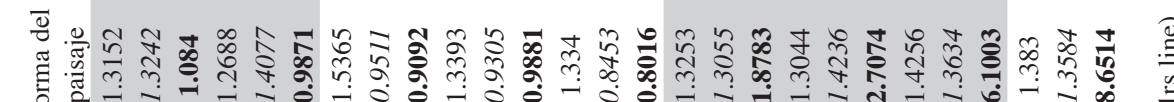

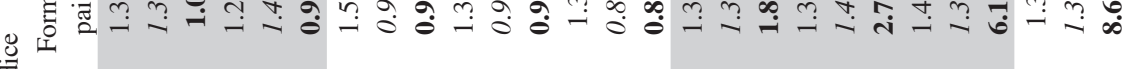

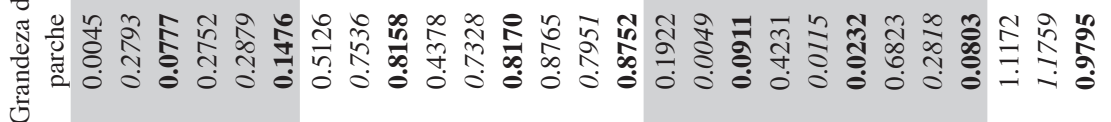

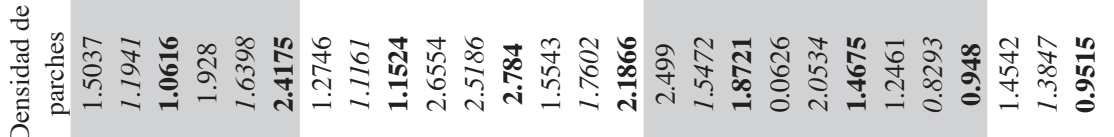

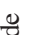

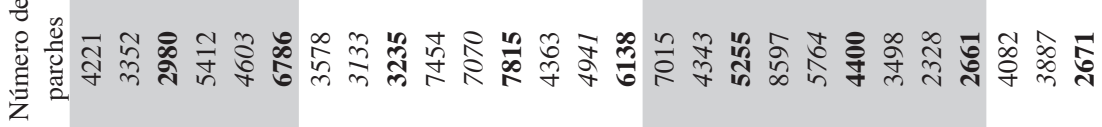

สँّ

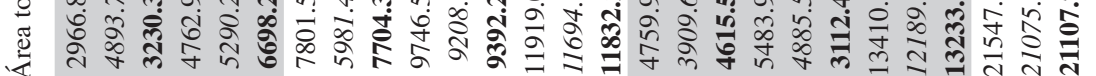

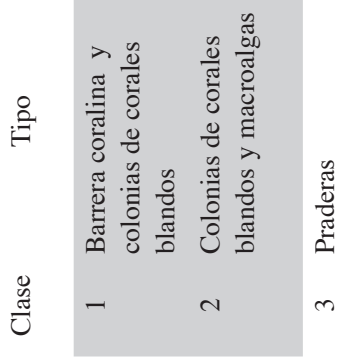

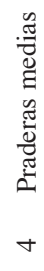

שֶ๊

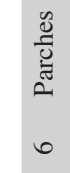

$\frac{0}{\frac{0}{0}}$

$\frac{}{\sqrt[3]{3}}$

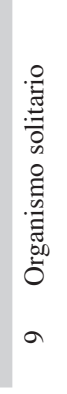

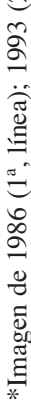




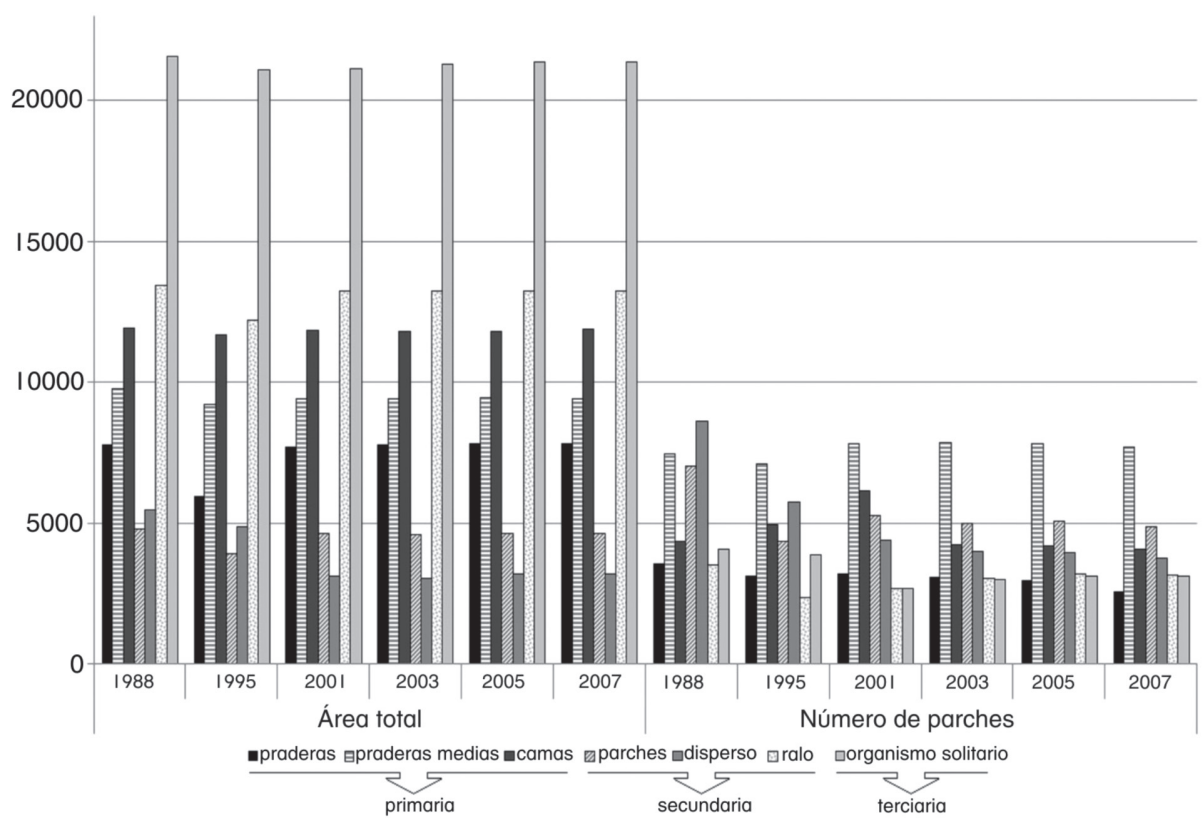

Fig. 5. Área total y número de parches en los distintos años y secciones que corresponden a los sitios de cobertura de T. testudinum en la bahía.

Fig. 5. Total area and patch numbers values for different years and sections of T. testudinum coverage sites in the bay.

en 1988 y sin signos de recuperación hasta 2007 , con aproximadamente $210 \mathrm{~km}^{2}$. De esta sección, las clases que se han recuperado a los cambios en el tiempo fueron la 6 y 8 , mientras que la 7 ha seguido perdiendo su cobertura (Cuadros 1 y 2, Fig. 5).

Esta sección ha registrado una disminución de su área total, lo que se ve reflejado en la cantidad de parches hasta el 2001, posteriormente se observa una estabilización de la cobertura, no así en el número de parches, pues se sigue observando una perdida. Respecto al análisis de 1986, las clases 6 y 8 presentan pérdidas menores a un $40 \%$ del área total de cada clase para 1988 y una mínima recuperación hasta $2007(11 \%)$. La clase 7 perdió un $10.91 \%$ en 1988 , un $43.24 \%$ para 2001 y un $44.90 \%$ para 2003 , por lo que a diferencia de la sección primaria, si existe una pérdida del área total de cobertura en un $12 \%$ hasta 2007 , que se corrobora con el índice de Grandeza del Parche que $=>0$, indicando un crecimiento nulo del área de los parches y donde los factores físicos (corrientes, sedimentación) terminan por desaparecer estos sitios y disminuyen el área total.

La tercera sección solo comprende a la clase 9 , que cubre aproximadamente $210 \mathrm{~km}^{2}$ y no presentó una pérdida espacial significativa de su cobertura (Cuadro 1; Fig. 5); para 1988 se había perdido $2.18 \%$, que en 2007 disminuyo solo un $0.89 \%$, se observa una pérdida total del área original de $1.29 \%$, que representó 0.97 de Índice de Tamaño del Parche, que índica crecimiento lento de los organismos solitarios en esta clase. La posición al interior de la bahía, el relativo aislamiento y protección por una isla de barrera, son condiciones que favorecen el bajo impacto por eventos, además de que los parches no son tan afectados, su número y el área total de la cobertura de T. testudinum se mantiene constante.

En general, el Índice de Forma del Paisaje (Fig. 6) refleja la baja fragmentación de las clases que corresponden al área principal, pues 


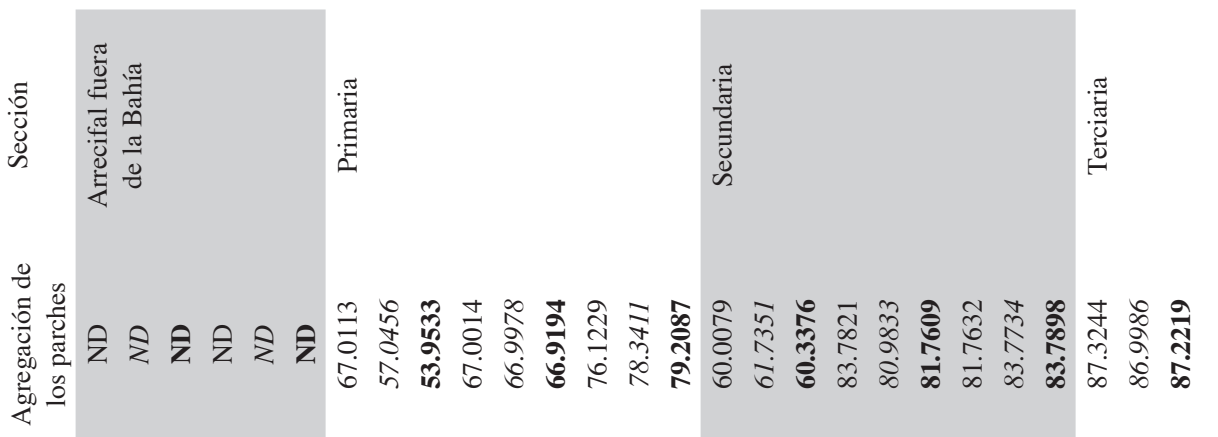

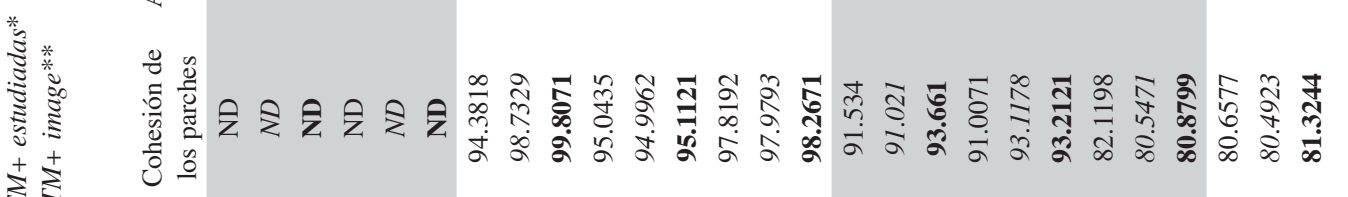

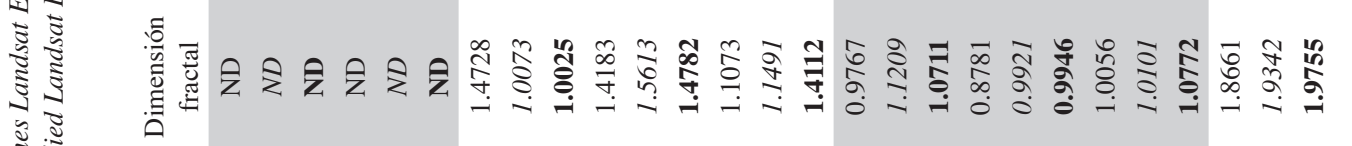
ปัฐ $\mathrm{d}$

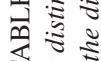
运安 部

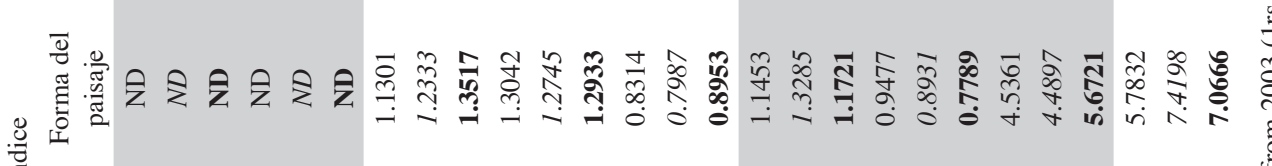

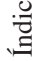

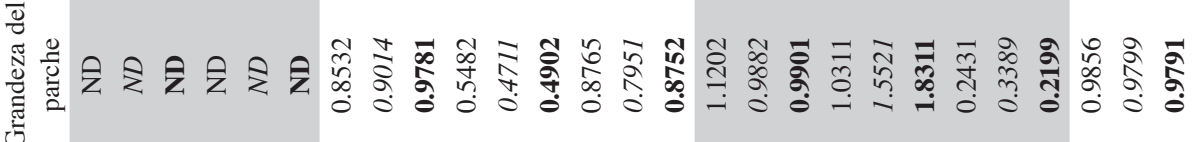
8

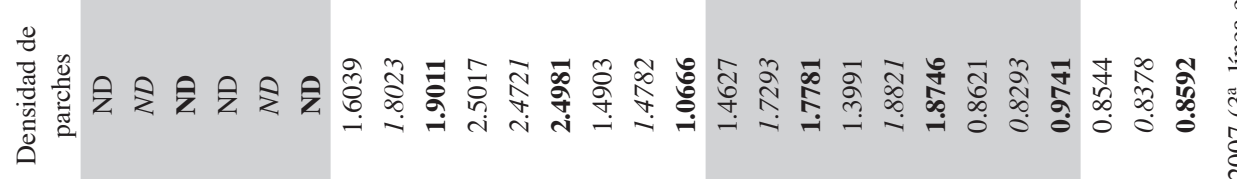

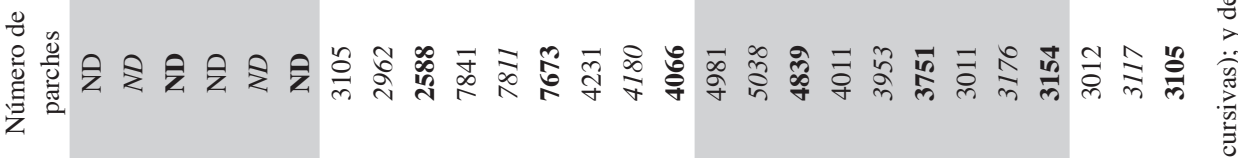
$\Xi+$

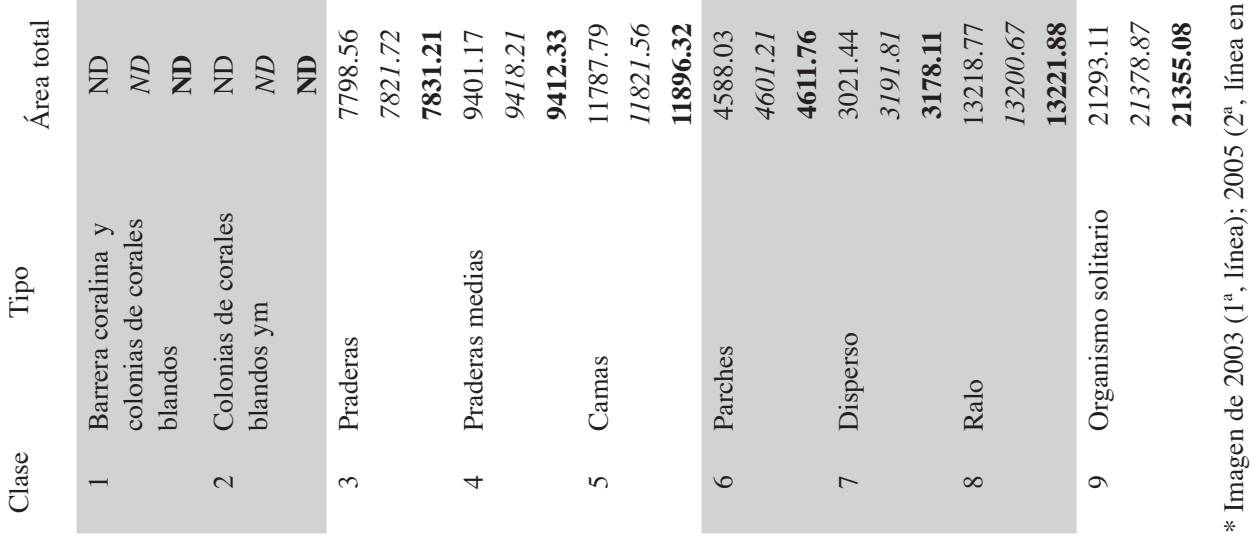




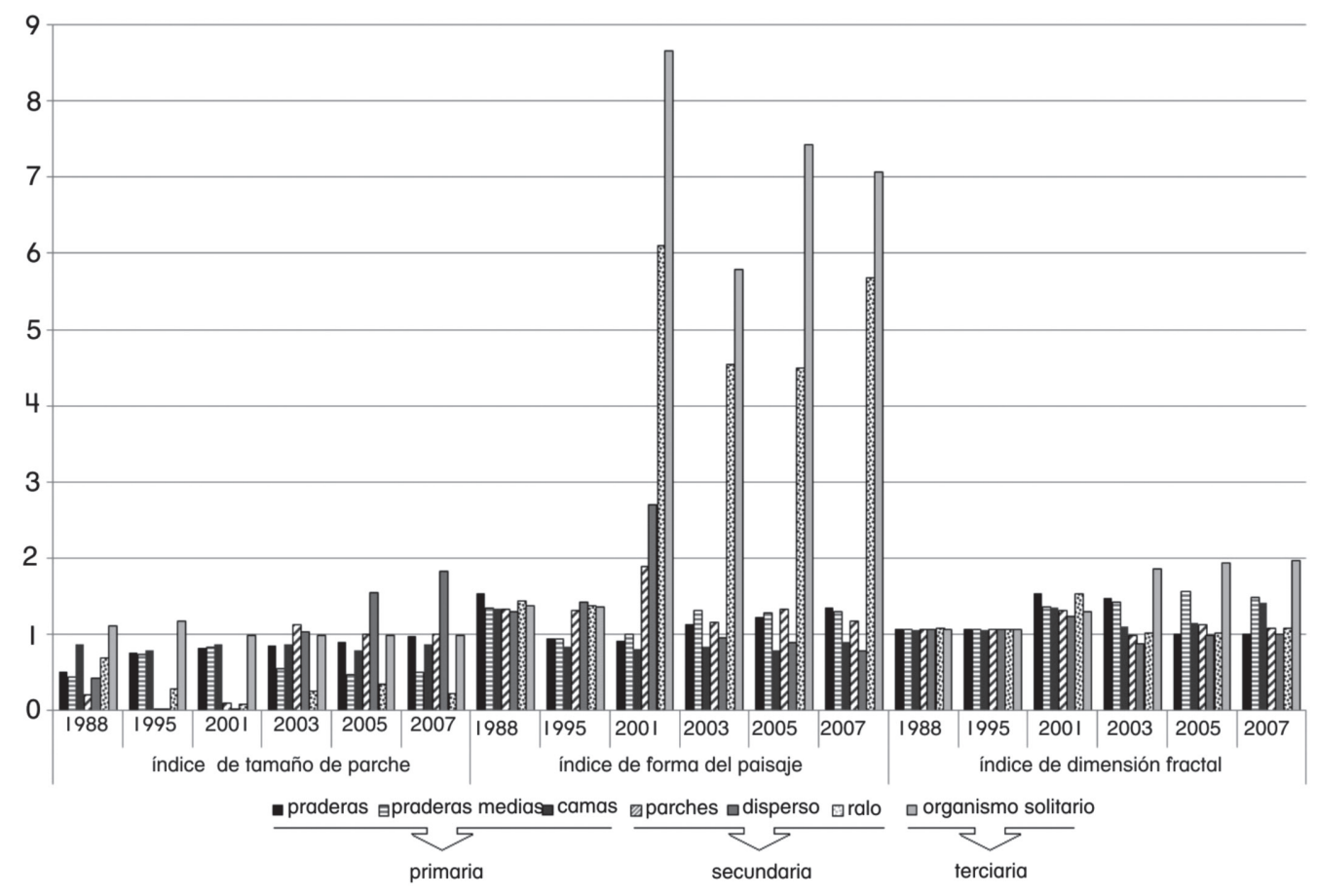

Fig. 6. Índices de tamaño del parche, forma del paisaje y dimensión fractal en los distintos años y secciones que corresponden a los sitios de cobertura de T. testudinum dentro de la bahía.

Fig. 6. Largest patch, shape and fractal dimension indexes for different years and sections of T. testudinum coverage sites in the bay.

se vieron valores reducidos, < a 1.6 en 1986 y < a 1 en 2001 (Cuadros 1 y 2). Sin embargo, en las áreas secundaria y terciaria se observó una alta fragmentación y pérdida de esos fragmentos, con valores del índice del Paisaje menores a 1.4 en 1986, incrementándose de forma significativa a un valor de 6.1 de la clase 8 en 2001 (Cuadro 1), siendo éste el valor más alto del área secundaria en los últimos años del análisis (2003 a 2007, Cuadro 2). El área terciaria presentó un incremento del valor del índice de 1.3 a 8.6 (Cuadro 1) y de 5.7 a 7.4 entre 2003 y 2005 (Cuadro 2), indicando una alta fragmentación.

La forma perimetral de estos parches está dada por el valor del Índice de Dimensión Fractal (Fig. 6), el cual indica que los valores obtenidos en las clases de la sección secundaria (clase 6, 7 y 8) son $>1$, e indican irregularidad del perímetro de los parches, mientras que el crecimiento en forma radial indica que los parches son jóvenes o que son objeto frecuente de impactos que alteran su perímetro y por ende su forma de crecimiento y expansión, a excepción de la clase 7 , cuyos valores fueron $<1$, lo que indican que son parches más irregulares y por lo tanto inmaduros, o que presentan una alta agregación de organismos (Cuadro 2). En la clase 9 se presentan los mayores valores del análisis, durante los últimos tres años del estudio, expresando mayor madurez de éstos fragmentos (Cuadro 2).

El Índice de Cohesión de los Parches (Fig. 7) revela que a pesar de existir una alta fragmentación de los parches de mayor tamaño, hay mucha conexión entre los mismos $(<90$ en 


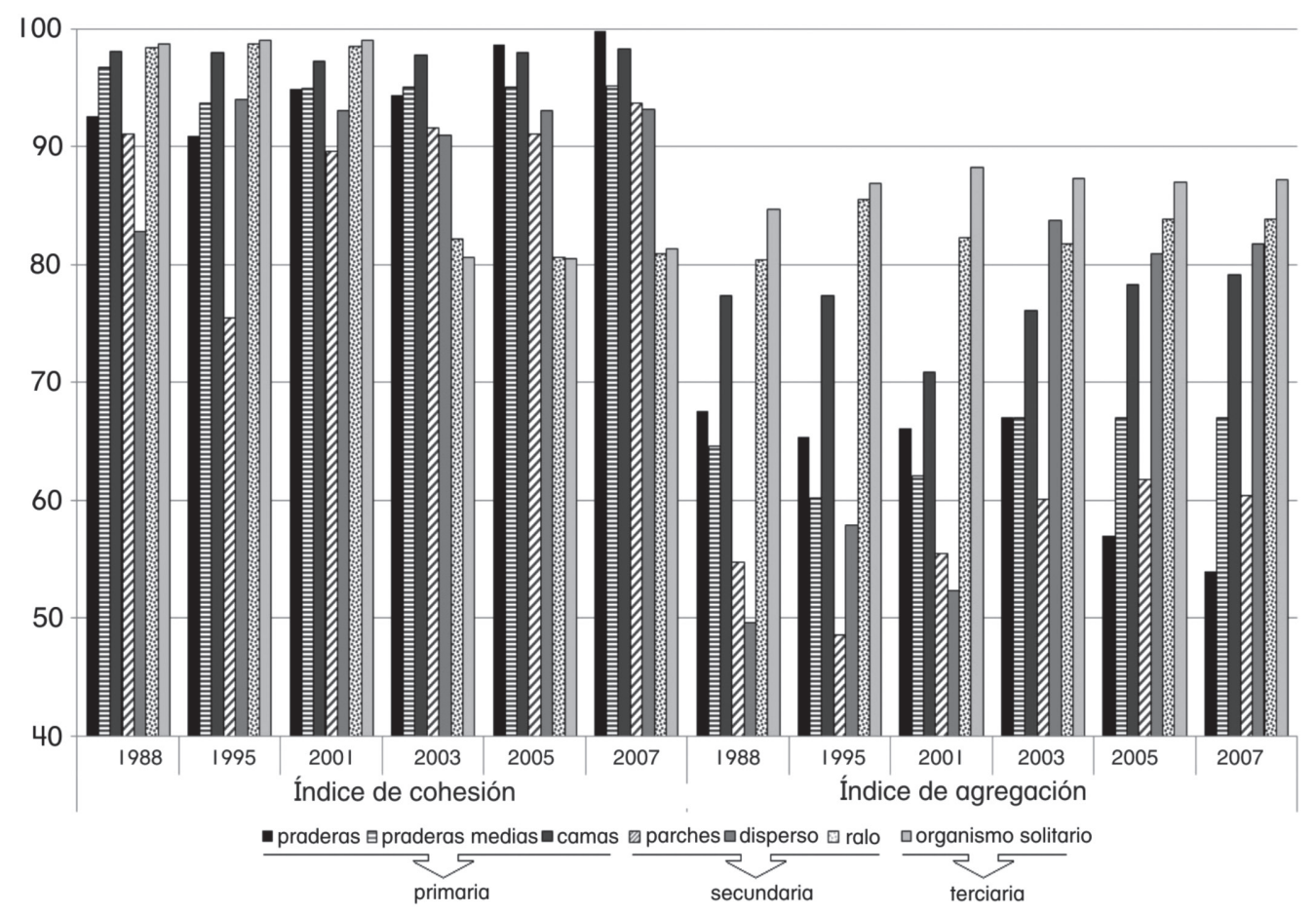

Fig. 7. Índices de cohesión y agregación de los parches en los distintos años y secciones que corresponden a los sitios de cobertura de T. testudinum dentro de la bahía.

Fig. 7. Cohesion and aggregation patch indexes for different years and sections of T. testudinum coverage sites in the bay.

la mayoría de los casos; Cuadros 1 y 2), donde las pequeñas particiones sirven como corredores ecológicos para las praderas de T. testudinum, y/o como corredores biológicos para las especies que ahí habitan, pues poseen una alta conectividad física. Las clases 8 y 9 presentan una mayor fragmentación en los tres últimos años del estudio (2003 a 2007), indicando una pérdida de dichos corredores biológicos (Cuadro 2).

Los altos porcentajes del Índice de Agregación de los Parches durante 1986, 1993 y 2001 (Cuadro 1, Fig. 7) en las clases 8 y 9, indican que los parches están altamente agregados en el área donde se encuentran, es decir, no hay un indicio de pérdida del área y de los parches, pero sin llegar a ser sitios compactos. Sin embargo, a partir del 2003 disminuyen los valores del índice, no así su área total, esto indica que los parches tienden a conectarse, lo que se corrobora con el Índice de Dimensión Fractal (Cuadro 2); las clases 6 y 7 presentan la mayor desagregación (ligeramente mayores al 50\%).

\section{DISCUSION}

Se han observado pérdidas de pastos marinos en el Caribe Mexicano (Cruz-Palacios \& van Tussenbroek 2005) debido a que están expuestas a eventos meteorológicos mayores como son las tormentas tropicales y los huracanes. Aunque se han presentado pérdidas de pastos marinos en otras lagunas costeras de la península de Yucatán, como en Celestún, Chelem, Nichupté y Bojórquez, estas han sido por actividades e impactos antrópicos, como son 
los sistemas de pesca artesanales (arrastres), el turismo en zonas someras y el aumento desmedido de la mancha urbana (Herrera-Silveira et al. 2000, Herrera-Silveira 2006). Sin embargo, Bahía de la Ascensión no presenta una infraestructura urbana o turística en su cuenca y se ha determinado en buen estado de conservación, Herrera-Silveira (2006) señaló que no se observan cambios significativos en la cobertura de pastos marinos al nivel de la composición de especies y relación pastos/macroalgas.

Sin embargo, las pérdidas de las praderas de pastos marinos en el margen costero del Caribe Mexicano en general, y en Bahía de la Ascensión en particular, como se observa en la presente investigación, fueron ocasionadas por los huracanes Gilberto y Roxanne, lo que tiene un impacto inmediato en las macrófitas enraizadas que incluye la defoliación, la exposición de su sistema de rizomas verticales (shoots), rizomas horizontales y raíces, hasta su total desarraigo (Cruz-Palacios \& van Tussenbroek 2005). Consecuentemente, con la resuspensión de los sedimentos hubo menor entrada de luz a la columna de agua y el material sedimentario enterró a la vegetación acuática sumergida creando la fragmentación de los parches como se observa en las clases 4 y 5 . Estos efectos han sido observados y cuantificados en las costas de Australia donde se han visto destruidos y segmentados cientos de kilómetros cuadrados de praderas de pastos marinos (Poiner et al. 1989, Preen et al. 1995), por el drástico decremento en la salinidad. Esto no sucede en Bahía de la Ascensión, se observa mayor resiliencia en las praderas de pastos marinos, pues se encuentran inmersas en un escenario ambiental de gradientes de salinidad amplios y porque su variabilidad natural le confiere mayor resistencia a estos eventos meteorológicos de gran escala, dándole mayor estabilidad ecológica a la vegetación acuática sumergida de este ecosistema (Arellano-Méndez 2004, Mumby et al. 2004, Johnson \& Marshall 2007).

La clase 7 presentó la mayor pérdida en su área a través del tiempo, esto puede asociarse a que al estar en una zona donde la profundidad del sedimento es menor, su textura es arenosa y dura por debajo (Mexicano-Cintora et al. 2003), por lo que las raíces y rizomas no penetran fácilmente, además la acción mecánica de la onda de marea de los huracanes facilitan desgarrar, arrancar y romper las estructuras aéreas y subterráneas de los pastos marinos (Michot et al. 2002). Adicionalmente, la escasa profundidad y la remoción de los sedimentos como un efecto del incremento en la turbulencia asociado al oleaje y las corrientes (Clarke \& Kirkman 1989, Short \& Neckles 1999, Arellano-Méndez 2004), deja al sistema de raíces y rizomas descubiertos, lo cual provoca su desarraigo y posterior desaparición, como se observó en Chandeleuer Sound, Lousiana durante el paso del huracán Camille (Posey \& Alphin 2002). Este efecto es mayor en sitios donde los sedimentos son más finos que los de Bahía de la Ascensión.

La fragmentación de las zonas donde se encuentran las clases con mayor cobertura (clases 3, 4 y 5), fue baja, a pesar de haber sido impactados por los huracanes. Los resultados de índices como el de sus fronteras (perímetro) indica buena recuperación gracias a su condición de organismos de larga vida y desarrollo clonal (Hemminga \& Duarte 2000), por lo que en dichas zonas podrían presentarse procesos que contribuyen a los patrones espaciales del paisaje (perdida y colonización natural, distribución de especies), los cuales requieren un análisis detallado sobre extensos intervalos de tiempo (Kendrick et al. 2002, 2008). Otros estudios sobre pastos marinos han sido conducidos con alta resolución, pero están limitados en cuanto a extensión (Robbins \& Bell 2000, Bell et al. 2006), dando un reflejo puntual de los efectos y no es comparable con toda la población o bien no evidencia los efectos integrales en áreas de gran tamaño, como BA.

Gran parte de la fragmentación de las praderas de $T$. testudinum ocurrida en Bahía de la Ascensión fue ocasionada tras el paso de los huracanes, el transporte de sedimentos que disminuye la cantidad de la luz en la columna de agua y aumento del material depositario (Michot et al. 2002, Byron \& Heck Jr. 2006, Steward et al. 2006, Cabaço et al. 2008) que 
entierra las praderas; esto se ha observado en la pérdida de praderas de Zostera marina en el Pacífico Mexicano debido a una significativa limitación de la luz por la resuspensión del material del fondo. Marba et al. (1994) estudiaron este efecto tras el paso del huracán Gilberto (septiembre 1988) en las praderas de T. testudinum en la costa del Caribe Mexicano, se observaron diferencias en la longitud internodal que se atribuyó al incremento en la depositación de sedimentos.

$\mathrm{El}$ aumento en las descargas de agua dulce como un producto de la lluvia contribuye al transporte de material orgánico y sedimentario hacia la costa (zonas de dunas, manglares), además de los aportes por descargas de agua subterránea en este tipo de ecosistemas con sustrato de naturaleza cárstica, lo que provoca reducción de la transparencia, salinidad y calidad del agua a corto plazo (Comín \& Herrera-Silveira 2000, Medina-Gómez \& Herrera-Silveira 2003, Steward et al. 2006), acelerando la usual pérdida invernal en la cobertura de pastos marinos. No se observó efecto a largo plazo, pues se registraron clases donde las praderas se recuperaron (Cuadro $1 \mathrm{y}$ 2), indicando buena condición de salud respecto a la calidad medio ambiental de la bahía, que podría estar favorecida por un bajo tiempo de residencia del agua y reducido cambio de uso del suelo en la cuenca (Herrera-Silveira 2006).

Este buen estado de salud se observa mejor en los tres últimos años del estudio, donde se identifica un efecto en la pérdida del número de parches, pero no se registra perdida en el área total de cobertura (Cuadro 2), indicando que los fragmentos se han unido, por lo que se pierden los corredores biológicos, pero ganando cobertura de áreas extensas, favorables para distintos organismos que usan estos ecosistemas de primera (se alimentan) o de segunda (vivienda, guardería o caza) fuente o como sistemas estabilizadores (Zieman 1975, McNeill \& Bell 1992). A escala del Gran Caribe se han registrado variaciones temporales parecidas en la biomasa total, productividad de hojas y tasa de renovación (Zieman 1986, Duarte 1989, CARICOMP 1997), el último registro que se tiene fue en 1999, en la Bahía de Chengue, Colombia (Rodríguez-Ramírez \& Garzón-Ferreira 2003), lugar caracterizado por su alta vitalidad y buen estado de conservación (Garzón-Ferreira \& Rodríguez-Ramírez 2003), al igual que BA.

Muchos de los hábitats de pastos marinos a nivel mundial han sido severamente dañados por efectos antrópicos o están experimentando un declive a nivel global (Lugo 2000, Duarte 2002, Paerl et al. 2006, Pielke Jr. et al. 2005, Steward et al. 2006, Orth et al. 2006 a y b). Los cambios hidrológicos y la eutrofización de los ecosistemas costeros podrían reducir la capacidad de recuperación de las praderas de pastos marinos debido a los efectos del CCG, por lo que la estrategia para mantener o recuperar la buena condición de salud de estas comunidades debe emprenderse tanto a nivel local, regional y global, se identificaron como acciones recomendadas: el saneamiento de las fuentes de agua dulce que llegan a los ecosistemas costeros, el manejo de residuos peligrosos y el mantenimiento de las características que favorecen la dinámica hidrológica natural de cada sistema.

Aunque el efecto del Cambio Climático Global sobre los huracanes aún no se comprende en su totalidad, éste puede afectar su velocidad máxima e intensidad (Lugo, 2000, Michot et al. 2002), provocando efectos en las praderas de pastos marinos, que son medibles a distintas escalas de tiempo y espacio, y que van desde acciones mecánicas, a efectos en la hidrología y calidad del agua.

Las variaciones observadas en los distintos Índices del Paisaje con respecto a la cobertura de T. testudinum en Bahía de la Ascensíon, indican gran resiliencia de la especie, que se recuperó tras el paso de huracanes de gran magnitud como fueron Gilberto y Roxanne $\mathrm{y}$ otros de menor magnitud y/o tormentas tropicales, que aunque no afectan de manera directa-mecánica las praderas, si pueden producir efectos a nivel fisiológico por los cambios temporales de corto-plazo (pulsos) de las condiciones hidrológicas. 
A pesar de que las praderas de pastos marinos han resistido eventos que podrían provocar una extinción, su futuro, igual que el de muchos ecosistemas costeros, se presenta sombrío, pues el calentamiento global provoca el incremento relativo del nivel medio del mar, períodos más largos con altas temperaturas del agua y mayor intensidad de las tormentas (Salazar-Vallejo 2002, Knowlton 2001), lo que reduce significativamente la capacidad de regeneración y crecimiento, o el mantener a la comunidad de pastos marinos en etapas seriales tempranas.

\section{AGRADECIMIENTOS}

Este trabajo es parte de la tesis doctoral de Leonardo Arellano-Méndez en el CINVESTAVIPN, U. Mérida. Este trabajo fue financiado con fondos de SISIERRA-CONACyT (Proyecto 20000706005), de los Laboratorios de Percepción Remota y SIG y de Producción Primaria del CINVESTAV-IPN U. Mérida y The Nature Conservancy (MxSomex0106070CINVETSAC-Pastos Marinos). Gracias al personal de la "Reserva de la Biosfera de Sian Ka'an" por las facilidades para el presente trabajo y en especial al grupo de estudiantes y auxiliares del laboratorio de Producción Primaria y el de Percepción Remota y SIG: Javier Ramírez, Jorge Acosta, Guadalupe Mexicano, Israel Medina, José Cámara, Octavio Cortés, Juan Asensio, Juan Camaal, Lucio Loman, Sara Morales y Elsy Alvarado por su ayuda en el campo y las actividades de laboratorio.

\section{RESUMEN}

Thalassia testudinum es la macrófita dominante en Bahía de la Ascensión (Quintana Roo, México), se estudió para conocer su distribución espacio-temporal y determinar si fue afectada por el paso de huracanes que se han visto incrementados en fuerza y frecuencia por el cambio climático. Partiendo de una imagen Landsat ETM+ de 2001 y usando información de grandes grupos morfofuncionales y de cobertura de T. testudinum adquiridas en 525 estaciones de muestreo, se obtienen las semillas para la clasificación supervisada de los hábitats bénticos (8 clases). Con el fin de cuantificar los cambios ocasionados por dos huracanes, se usaron dos imágenes, una de 1988 (Gilberto) y 1995 (Roxanne) y tres más (2003, 2005 y 2007) para monitoreo sin efectos meteorológicos mayores; a estos 6 mapas categóricos se les aplicó un análisis de Ecología del Paisaje usando 8 índices que describen las características espaciales, de estructura, función, cambio de los elementos (matriz-mancha-corredor), efectos sobre el ecosistema, conectividad, bordes, forma del parche y fragmentación del hábitat. Los modelos indican que T. testudinum puede clasificarse como un continuo (matriz), pues los fragmentos no se observan de forma intermitente, sino como una progresión de zonas mínimas a máximas en referencia a su cobertura (corredores ecológicos). No poseen una forma regular, indicando que los impactos son recientes y pueden ser debidos a efectos directos (huracanes de alta intensidad) e indirectos (aporte de sedimentos). Los fragmentos de tipo "suelos desnudos" presentan una distribución discontinua, considerándose sitios que han permanecido estables en una larga escala de tiempo; las zonas con coberturas más densas ("camas", "praderas medias" y "praderas") presentan baja fragmentación y alta conexión de sus fragmentos. Las características de poseer un perímetro irregular y crecimiento de formal radial, sugieren que el impacto de los meteoros no tiene efecto en la capacidad de resiliencia de T. testudinum en este ecosistema, indicando el buen estado de la calidad ambiental de la bahía para su crecimiento.

Palabras clave: Thalassia testudinum, cambio climático global, huracanes, ecología del paisaje, resiliencia, índices, fragmentación, cobertura.

\section{REFERENCIAS}

Aguayo, C.M. 2004. Estructura de la vegetación acuática sumergida como bioindicador de la calidad del agua en una zona costera tropical. Tesis de Maestría, Centro de Investigación y de Estudios Avanzados del Instituto Politécnico Nacional (CINVESTAV-IPN), Unidad Mérida, Yucatán, México.

Aguilar, M.A., L.E. Aguilar-Rosas \& J.A. Fernández. 1989. Algas marinas bentónicas de la Bahía de la Ascención, Quintana Roo, México. Bol. Inst. Oceanogr. 28: 67-75.

Aranda, C.N. 2001. Alimentando al mundo, envenenando al planeta: eutrofización y calidad del agua. Avance y Perspectiva 20: 293-303.

Arellano-Méndez, L.U. 2004. Análisis espacio-temporal de las variables hidrológicas: detección de heterogeneidad a gran escala temporal. Tesis de Maestría, Centro de Investigación y de Estudios Avanzados del Instituto Politécnico Nacional (CINVESTAV-IPN), Unidad Mérida, Yucatán, México. 
Bell, S.S., M.S. Fonseca \& N.B. Stafford. 2006. Seagrass ecology: new contributions from a landscape perspective, p. 625-645. In A.W.D. Larkum, R.J. Orth \& C.M. Duarte (eds.). Seagrasses: biology, ecology and conservation. Springer, Dordrecht, Holanda.

Byron, D. \& K.L. Heck Jr. 2006. Hurricanes effects on seagrasses along Alabama's Gulf. Estuar. Coast. 29: 939-942.

Cabaço, S., R. Santos \& C.M. Duarte. 2008. The impact of sediment burial and erosion on seagrasses: A review. Estuar. Coast. Shelf S. 79: 354-366.

CARICOMP. 1997. Variation in ecological parameters of Thalassia testudinum across the CARICOMP network. Proc. 8th Int. Coral Reef Symp. 1: 663-668.

Clarke, S.M. \& H. Kirkman. 1989. Seagrass dynamics, p. 304-345. In A.W.D. Larkim, A.J. McComb \& S.A. Shepherd. Biology of seagrasses. Elsevier, Amsterdam, Holanda.

Comín, F.A. \& J.A. Herrera-Silveira. 2000. The role of birds on the trophic structure and nutrient cycles of aquatic ecosystems: a review. In F.A. Comín, J.A. Herrera-Silveira \& J. Ramírez-Ramírez (eds.). Proceedings, Limnology and Waterfowl, Monitoring, Modeling and Management, Workshop, Aquatic Birds Working Group. Societas Internationalis Liknologine, Universidad Autónoma de Yucatán. Mérida, Yucatán, México.

Cruz-Palacios, V. \& B.I. van Tussenbroek. 2005. Simulation of hurricane-like disturbances on a Caribbean seagrass bed. J. Exp. Mar. Biol. Ecol. 324: 44-60.

Dolbeth, M., P.G. Cardoso, S.M. Ferreira, T. Verdelhos, D. Raffaelli \& M.A. Pardal. 2007. Anthropogenic and natural disturbance effects on a macrobenthic estuarine community over a 10-year period. Mar. Pollut. Bull. 54: 576-585.

Duarte, C.M. 1989. Temporal biomass variability and production/biomass relationships of sea grass communities. Mar. Ecol. Prog. Ser. 51: 269-276.

Duarte, C.M. 2002. The future of seagrass meadows. Environ. Conserv. 29: 192-206.

Emanuel, K.A. 2005. Increasing destructiveness of tropical cyclons over the past 30 years. Nature 436: 686-688.

Fourqurean, J.W., J.N. Boyer, M.J. Durako, L.N. Hefty \& B.J. Peterson. 2003. Forecasting response of seagrass distribution to changing water quality using monitoring data. Ecol. Appl. 13: 474-489.
Garzón-Ferreira, J. \& A. Rodríguez-Ramírez. 2003. Diez años de monitoreo en una pradera de Thalassia, 71-74. p. 159. In Díaz, J.M., L.M. Barrios \& D.I. Gómez-López. Las praderas de pastos marinos en Colombia: Estructura y distribución de un ecosistema estratégico. INVEMAR, Serie de Publicaciones Especiales No. 10, Santa Marta, Colombia.

Heck Jr., K.L. \& D. Byron. 2005. Post-Hurricane Ivan damage assessment of seagrass resources of coastal Alabama. Dauphin Island Sea Lab report, Dauphin Island, Alabama, EEUU.

Hemminga, M.A. \& C.M. Duarte. 2000. Seagrass Ecology. Cambridge, Cambridge, Inglaterra.

Herrera-Silveira, J.A. 2006. Lagunas Costeras de Yucatán (SE, México). Ecotrópicos 19: 94-108.

Herrera-Silveira, J.A., J. Ramírez-Ramírez, N. Gómez \& A. Zaldivar. 2000. Seagrass bed recovery after hydrological restoration in a costal lagoon with groundwater discharges in the North of Yucatán, p. 123-135. In S. Bortone (ed.). Seagrass: monitoring ecology, physiology and management. CRC, Boca Raton, Florida, EEUU.

Johnson, J.E. \& P.A. Marshall. 2007. Climate change and the Great Barrier Reef: a vulnerability assessment. Great Barrier Reef Marine Park Authority, Townsville, Australia.

Kendrick, G.A., M.J. Aylward, B.J. Hegge, M.L. Cambridge, K. Hillman, A. Wyllie \& D.A. Lord. 2002. Changes in seagrass coverage in Cockburn Sound, Western Australia between 1967 and 1999. Aquat. Bot. 73: 75-87.

Kendrick, G.A., K.W. Holmes \& K.P. van Niel. 2008. Multi-scale spatial patterns of three seagrass species with different growth dynamics. Ecography 31: 191-200.

Kennish, M.J. 2002. Environmental threats and environmental futures of estuaries. Environ. Conserv. 29: 78-107.

Knowlton, N. 2001. Coral reef biodiversity-habitat size matters. Science 292: 1493-1495.

Liceaga-Correa, M.A. 2003. Reporte Final. Caracterización ecológica, actual y retrospectiva, de los hábitats bénticos y su relación con la pesquería de la langosta Panulirus argus. Bahía de la Ascensión, Reserva de la Biosfera de Sian Ka'an. Proyecto SISIERRA 20000706005.

Lillebø, A.I., J.M. Neto, I. Martins, T. Verdelhos, S. Leston, P.G. Cardoso, S.M. Ferreira, J.C. Marques \& M.A. Pardal. 2005. Management of a shallow temperate 
estuary to control eutrophication: the effect of hydrodynamics on the system's nutrient loading. Estuar. Coast. Shelf S. 65: 697-707.

Lugo, A.E. 2000. Effects and outcomes of Caribbean hurricanes in a climate change scenario. Sci. Total Environ. 262: 243-251.

Marba, N., M.E. Gallegos, M. Merino \& C.M. Duarte. 1994. Vertical growth of Thalassia testudinum: seasonal and interannual variability. Aquat. Bot. 47: 1-11.

McGarigal, K., S.A. Cushman, M.C. Neel \& E. Ene. 2002. FRAGSTATS: spatial pattern analysis program for categorical maps. Universidad de Massachusetts, Amherst, EEUU. (Disponible en línea: http://www. umass.edu/landeco/research/fragstats/fragstats.html)

McNeill, S.E. \& J.D. Bell. 1992. Comparison of beam trawls for sampling macrofauna of Posidonia seagrass. Estuaries 15: 360-367.

Medina-Gómez, I. \& J.A. Herrera-Silveira. 2003. Spatial characterization of water quality in a Karstic coastal lagoon without anthropogenic disturbance: a multivariate approach. Estuar. Coast. Shelf S. 58: 455-465.

Mexicano-Cintora, G., M.A. Licega-Correa, L.U. ArellanoMéndez \& H. Hernández-Núñez. 2003. Caracterización espacial y sistemática de los sedimentos superficiales de Bahía de la Ascensión, Quintana Roo. Congreso Nacional de Ciencia y Tecnología del Mar, Boca del Río, Veracruz, México.

Michot, T.C., J.N. Burch, A. Arrivillaga, P.S. Rafferty, T.W. Doyle \& R.S. Kemerer. 2002. Impacts of Hurricane Mitch on seagrass beds and associated shallow reef communities along the Caribbean coast of Honduras and Guatemala. U.S. Geological Survey Open File Report 03-181, National Wetlands Research Center, Lafayette, Louisiana, EEUU.

Milner, C. \& R.E. Hughes. 1968. Methods for the measurement of the primary production of seagrasses. Grassland-IBP Handbook $N^{\circ}$ 6, Blackwell, Londres, Inglaterra.

Mumby, P.J., A.J. Edwards, J.E. Arias-Gonzalez, K.C. Lindeman, P.G. Blackwell, A. Gall, M.I. Gorczynska, A.R. Harborne, C.L. Pescod, H. Renken, C.C. Wabnitz \& G. Llewellyn. 2004. Mangroves enhance the biomass of coral reef fish communities in the Caribbean. Nature 427: 533-536.

Orth, R.J., T.J.B. Carruthers, W.C. Dennison, C.M. Duarte, J.W. Fourqurean, K.L. Heck, A.R. Hughes, G.A. Kendrick, W.J. Kenworthy, S. Olyarnik, F.T. Short, M. Waycott \& S.L. Williams. 2006a. A global crisis for seagrass ecosystems. BioScience 56: 987-996.
Orth, R.J., M.L. Luckenbach, S.R. Marion, K.A. Moore \& D.J. Wilcox. 2006b. Seagrass recovery in the Delmarva coastal bays. Aquat. Bot. 84: 26-36.

Paerl, H.W. 2006. Assessing and managing nutrient-enhanced eutrophication in estuarine and coastal waters: interactive effects of human and climatic perturbations. Ecol. Eng. 26: 40-54.

Paerl, H.W., L.M. Valdes, J.E. Adolf, B.M. Peierls \& L.W. Harding Jr. 2006. Anthropogenic and climatic influences on the eutrophication of large estuarine ecosystems. Limnol. Oceanogr. 5: 448-462.

Pielke Jr. R.A., C. Landsea, K. Emanuel, M. Mayfield, J. Laver \& R. Pasch. 2005. In press. Hurricanes and Global Warming. Bulletin of the American Meteorological Society. (Disponible en línea : http://sciencepolicy. colorado.edu/admin/publication_files/resourse-1762hurricanes\%20and_global_warming.pdf)

Poiner, I.R., D.I. Walker \& R.G. Coles. 1989. Regional studies seagrasses of tropical Australia, p. 279-303. In A.W. Larkum, A.J. McComb \& S.A. Sheperd (eds.). Biology of seagrasses. Elsevier, Amsterdam, Países Bajos.

Posey, M. \& T. Alphin. 2002. Resilience and stability in an offshore benthic community: responses to sediment borrow activities and hurricane disturbance. J. Coastal Res. 18: 685-697.

Preen, A.R., W.J. Lee-Long \& R.G. Coles. 1995. Flood and cyclone related loss, and partial recovery, of more than $100 \mathrm{~km} 2$ of seagrass in Hervey Bay Queensland, Australia. Aquat. Bot. 52: 3-17.

Robbins, B.D. \& S.S. Bell. 2000. Dynamics of a subtidal seagrass landscape: seasonal and annual change in relation to water depth. Ecology 81: 1193-1205.

Rodríguez-Ramírez, A. \& J. Garzón-Ferreira. 2003. Monitoreo de arrecifes coralinos, pastos marinos y manglares en la Bahía de Chengue (Caribe colombiano). INVEMAR, Serie de Publicaciones Especiales No. 8, Santa Marta, Colombia.

Salazar-Vallejo, S.I. 2002. Huracanes y biodiversidad costera tropical. Rev. Biol. Trop. 50: 415-428.

Sheik, P.A. 2005. The impact of Hurricane Katrina on biological resources. Congressional Research Service Report for Congress. Order Code RL33117 (Disponible en línea: http://www.ncseonline.org/NLE/CRS/).

Short, F.T. \& H.A. Neckles. 1999. The effects of global climate change on seagrasses. Aquat. Bot. 63: 169-196. 
Steward, J.S., R.W. Virnstein, M.A. Lasi, L.J. Morris, J.D. Miller, L.M. Hall \& W.A. Tweedale. 2006. The impacts of the 2004 hurricanes on hydrology, water quality, and seagrass in the Central Indian River Lagoon, Florida. Estuar. Coast. 29: 954-965.

Webster, P.J., G.J. Holland, J.A. Curry \& H.R. Chang. 2005. Changes in tropical cyclone number, duration and intensity in a warming environment. Science 309 : 1844-1846.

Zieman, J.C. 1975. Tropical seagrass ecosystems and pollution, p. 63-74. In E.J. Ferguson \& E.J. Johannes (eds.). Tropical Marine Pollution. Elsevier, Amsterdam, Holanda.
Zieman, J.C. 1982. The ecology of seagrasses of south Florida: a community profile. Office of Biological Services. FWS/OBS-82/ 25. Fish and Wildlife Services, Washington D.C., EEUU.

Zieman, J.C. 1986. Gradients in Caribbean seagrass ecosystems, p. 25-29. In J.C. Ogden \& E.H. Gladfelter (eds.). Caribbean coastal marine productivity. Unesco, Reports in Marine Science.

Zieman, J.C., J.W. Fourqurean \& A.A. Frankovich. 1999. Seagrass die-off in Florida Bay: long-term trends in abundance and growth of turtle grass Thalassia testudinum. Estuaries 22: 460-470. 